\title{
Three- and Four-Nucleon Systems from Chiral Effective Field Theory
}

\author{
E. Epelbaum, ${ }^{1}$ H. Kamada, ${ }^{1,2}$ A. Nogga,${ }^{2}$ H. Witała, ${ }^{3}$ W. Glöckle, ${ }^{2}$ and Ulf-G. Meißner ${ }^{1}$ \\ ${ }^{1}$ Forschungszentrum Jülich, Institut für Kernphysik (Theorie), D-52425 Jülich, Germany \\ ${ }^{2}$ Institut für Theoretische Physik II, Ruhr-Universität Bochum, D-44780 Bochum, Germany \\ ${ }^{3}$ Institute of Physics, Jagellonian University, PL-30059 Cracow, Poland
}

(Received 26 July 2000)

\begin{abstract}
Recently developed chiral nucleon-nucleon $(N N)$ forces at next-to-leading order (NLO), that describe $N N$ phase shifts up to about $100 \mathrm{MeV}$ fairly well, have been applied to $3 N$ and $4 N$ systems. FaddeevYakubovsky equations have been solved rigorously. The resulting $3 N$ and $4 N$ binding energies are in the same range as found using standard $N N$ potentials. In addition, low-energy $3 N$ scattering observables are very well reproduced as for standard $N N$ forces. The long-standing $A_{y}$ puzzle is absent at NLO. The cutoff dependence of the scattering observables is rather weak.
\end{abstract}

DOI: $10.1103 /$ PhysRevLett.86.4787

Effective field theory (EFT) has become a standard tool in modern physics and is applied to a large variety of systems. It can also be used to construct nuclear forces in a systematic and controlled manner. The spontaneously and explicitly broken chiral symmetry of QCD can be implemented in the EFT formulated in terms of the asymptotically observed Goldstone boson (pion) and matter (nucleon) fields. In the purely pionic and the pion-nucleon systems, there is an expansion parameter which is a typical external momentum divided by a hadronic mass scale of the order of $1 \mathrm{GeV}$. Any $S$-matrix element can be systematically expanded in terms of this small parameter based on a power counting. In systems with more than one nucleon, an additional nonperturbative resummation is mandatory to deal with the shallow nuclear bound states. This idea was put forward by Weinberg [1] and taken up by van Kolck et al. [2,3] in the construction of two-nucleon $(N N)$ and three-nucleon $(3 N)$ forces. One basically constructs a potential based on the power counting and calculates bound and scattering states by use of a properly regularized Lippmann-Schwinger or Schrödinger equation. One outstanding result was that $3 \mathrm{~N}$ forces (3NF) vanish to leading order [1]. Other groups also investigated lowenergy properties of $N N$ systems along these lines [4,5]. A different counting scheme was proposed by Kaplan et al. [6] (KSW) working directly with the scattering amplitudes. Another important feature which distinguishes the KSW approach from Weinberg's is the perturbative treatment of the one-pion exchange (OPE). It has recently been shown that the KSW framework does not converge in the triplet waves for momenta larger than half of the pion mass [7]. In any case, this type of framework for the first time offers the possibility of calculating nuclear forces directly from fundamental principles and has a direct link to the chiral properties of QCD. Furthermore, this approach is firmly based on quantum field theory and avoids ill-defined concepts such as meson-nucleon form factors. In [8] we have taken up Weinberg's idea and constructed a $N N$ and $3 N$ potential based on the most
PACS numbers: $21.45 .+\mathrm{v}, 21.30 .-\mathrm{x}, 25.10 .+\mathrm{s}, 27.10 .+\mathrm{h}$

general effective chiral pion-nucleon Lagrangian using the method of unitary transformations. In this method the field theoretical pion-nucleon Hamiltonian is decoupled such that an effective purely nucleonic Hamiltonian consistent with a power counting scheme arises. In our formalism, we arrive at Hermitian energy-independent nuclear forces which we consider to be a major advantage with respect to applications to $3 N$ and $4 N$ systems, the issue of this Letter. In $[1,3,8,9] N N$ forces have been developed at leading, next-to-leading, and next-to-next-to-leading orders, LO, NLO, and NNLO, respectively. At LO the potential is represented by the ordinary OPE (with a pointlike coupling) as well as two contact interactions without derivatives. At NLO one includes the leading chiral two-pion exchange (TPE) as well as all possible contact interactions with two derivatives, whereas at NNLO we have additional TPE with low-energy constants (LECs) determined from pion-nucleon scattering [10]. The forces are properly renormalized and contain nine parameters related to those four-fermion contact terms. The OPE and TPE pieces are parameter free. The nine LECs have been uniquely fixed to low-energy $N N$ phase shifts in the $s$ and $p$ waves. The parameter free predictions for higher energies and partial waves and also deuteron properties are in general rather good. It was also observed that the NNLO predictions are better than the ones based on the NLO potential, as expected in a systematic EFT.

The natural question arises now, whether the $N N$ forces based on chiral perturbation theory ( $\chi \mathrm{PT}$ ) will be also successful in describing $3 N$ and $4 N$ low-energy observables. To that aim we solve the Faddeev-Yakubovsky equations rigorously for $3 N$ and $4 N$ systems [11-13] and determine binding energies and various scattering observables. To the best of our knowledge this is the first time that $\chi \mathrm{PT}$ has been practically applied to nuclear systems beyond $A=2$ within the Hamiltonian approach. In this first application we restrict ourselves to the NLO NN potential. In a forthcoming article we shall go on to NNLO and include also $3 \mathrm{NFs}$, which occur at that order the first time. The NLO 
results presented here are therefore parameter free and can serve as a good testing ground for the usefulness of the approach. Of course, some aspects of the $3 N$ system have already been studied in nuclear EFT $[14,15]$, based on a different counting and not including nonperturbative pions, which we consider a crucial ingredient if one wants to achieve an overall good description on the $N N$ and the multinucleon systems up to pion production threshold.

In order to use the chiral $N N$ forces in the $N N$ Lippmann-Schwinger equation one has to introduce a momentum regulator $\Lambda$. We remark that this regularization on the level of the scattering equation is completely different from standard methods which are applied to individual diagrams. Here we use the smooth regulator; its precise form is given in [9]. In order to investigate the cutoff dependence of $3 N$ and $4 N$ observables we have generated several $N N$ potentials corresponding to different cutoffs between $\Lambda=540$ and $600 \mathrm{MeV} / c$. They were all fitted to the ${ }^{1} S_{0},{ }^{3} S_{1}{ }^{3} D_{1},{ }^{1} P_{1}$, and ${ }^{3} P_{0,1,2} N N$ phase shifts up to $E_{\text {lab }}=100 \mathrm{MeV}$ [16]. In [9] we had already demonstrated that going to higher order in the EFT reduces the cutoff dependence and allows us to choose larger values for the cutoffs, as expected from general arguments [17]. The resulting phase shifts for NLO agree fairly well with the Nijmegen phase shift analysis (PSA) [18] and the ones obtained from the CD-Bonn potential [19]; see Table I. We know from [9] that one has to go to NNLO to improve systematically on that. Also, we restrict ourselves to the isospin symmetric case. Thus we do not take into account various charge independence and charge symmetry breaking effects such as, e.g., the pion mass difference, which are known to be significant at very low energies. Such effects can also be included systematically in our EFT [20].

Let us regard $3 N$ and $4 N$ binding energies now. We find for the fully converged solutions the $3 N$ and $4 N$ binding energies as given in Table II. The ranges are compatible with what is found using realistic potentials [11]. Note that the $N N$ forces were included up to the total $N N$ angular momentum of $j_{\max }=6$. All predicted binding energies are within $10 \%$ and $20 \%$ off the experimental values for ${ }^{3} \mathrm{H}$ and ${ }^{4} \mathrm{He}$, respectively. The somewhat large deviation in the case of ${ }^{4} \mathrm{He}$ indicates the importance of NNLO effects.

Also $3 \mathrm{~N}$ scattering can be solved rigorously nowadays [21] and we show in Figs. 1 and 2 a small selection out of the great wealth of observables in comparison to data and the theoretical predictions of CD-Bonn. Three energies, one below the $n d$ breakup threshold and two above, are chosen. Note further that we do not indicate error bars for the data since in most cases they will not be distinguishable on this scale. In all cases we compare the predictions of the chiral $N N$ potentials for the cutoffs $\Lambda=540$ and $\Lambda=600 \mathrm{MeV} / c$ to the ones of the CD-Bonn. As the simplest observable we show first the $n d$ total cross section in Fig. 1(a). The three theoretical curves overlap completely
TABLE I. Neutron-proton phase shifts in our approach (upper row) compared to the Nijmegen PSA (middle row) and the CD-Bonn potential (lower row).

\begin{tabular}{|c|c|c|c|c|}
\hline & $1 \mathrm{MeV}$ & $5 \mathrm{MeV}$ & $10 \mathrm{MeV}$ & $20 \mathrm{MeV}$ \\
\hline \multirow{3}{*}{${ }^{1} S_{0}$} & 62.044 & 63.869 & 60.28 & 53.76 \\
\hline & 62.078 & 63.645 & 59.97 & 53.56 \\
\hline & 62.069 & 63.627 & 59.96 & 53.57 \\
\hline \multirow{3}{*}{${ }^{3} S_{1}$} & 147.695 & 118.308 & 102.87 & 86.33 \\
\hline & 147.748 & 118.175 & 102.60 & 86.09 \\
\hline & 147.747 & 118.178 & 102.61 & 86.12 \\
\hline \multirow{3}{*}{$\epsilon_{1}$} & 0.107 & 0.679 & 1.17 & 1.65 \\
\hline & 0.105 & 0.674 & 1.16 & 1.66 \\
\hline & 0.105 & 0.672 & 1.16 & 1.66 \\
\hline \multirow{3}{*}{${ }^{3} D_{1}$} & -0.005 & -0.181 & -0.67 & -2.07 \\
\hline & -0.005 & -0.184 & -0.68 & -2.06 \\
\hline & -0.005 & -0.183 & -0.68 & -2.05 \\
\hline \multirow{3}{*}{${ }^{1} P_{1}$} & -0.187 & -1.493 & -3.08 & -5.54 \\
\hline & -0.189 & -1.503 & -3.08 & -5.47 \\
\hline & -0.187 & -1.487 & -3.04 & -5.40 \\
\hline \multirow{3}{*}{${ }^{3} P_{0}$} & 0.187 & 1.676 & 3.73 & 7.06 \\
\hline & 0.177 & 1.608 & 3.62 & 6.92 \\
\hline & 0.180 & 1.626 & 3.65 & 6.95 \\
\hline \multirow{3}{*}{${ }^{3} P_{1}$} & -0.117 & -0.994 & -2.16 & -4.18 \\
\hline & -0.108 & -0.932 & -2.05 & -4.01 \\
\hline & -0.108 & -0.937 & -2.06 & -4.03 \\
\hline \multirow{3}{*}{${ }^{3} P_{2}$} & 0.020 & 0.237 & 0.70 & 2.05 \\
\hline & 0.022 & 0.255 & 0.72 & 1.84 \\
\hline & 0.022 & 0.251 & 0.71 & 1.84 \\
\hline
\end{tabular}

at very low energies and then the chiral predictions start to deviate somewhat from the data as expected for our EFT at NLO.

In case of the $3 N$ breakup we selected in Figs. 1(b)1(d) a few often investigated configurations: the space star, a final state interaction peak configuration, and a quasifree scattering (QFS) configuration, respectively. We find very good agreement of the chiral $N N$ force predictions with the one from CD-Bonn. In case of QFS 1(d) and space star 1(b) some of the discrepancies are expected to be caused by Coulomb force effects not included in the theory. The upper group of data in 1(b) is $n d$ data and the disagreement with the theory presents a well-known puzzle at the moment [26].

For elastic $n d$ scattering we display in Fig. 2 the angular distributions, the nucleon analyzing power $A_{y}$, and the tensor analyzing powers $T_{2 k}$. Except for $A_{y}$ there are no $n d$ data for those energies. The discrepancies between data and theory for $T_{2 k}$ and for the differential cross section can be traced back to $p p$ Coulomb force effects [33]. Thus except for $A_{y}$ the agreement of CD-Bonn (thick solid curve) with the data is rather good, which is a well-known fact and is just given for the sake of orientation. The dotted and dashed curves are the chiral $N N$ force predictions 
TABLE II. Theoretical ${ }^{3} \mathrm{H}$ and ${ }^{4} \mathrm{He}$ binding energies for different cutoffs $\Lambda$ compared to CD-Bonn predictions and to the experimental ${ }^{3} \mathrm{H}$ binding energy and the Coulomb corrected ${ }^{4} \mathrm{He}$ binding energy. The kinetic energies and $S^{\prime}, P$, and $D$ state probabilities for ${ }^{4} \mathrm{He}$ are also shown.

\begin{tabular}{ccccccr}
\hline \hline Potential & $E_{T}(\mathrm{MeV})$ & $E_{{ }^{4} \mathrm{He}}$ & $T(\mathrm{MeV})$ & $S^{\prime}(\%)$ & $P(\%)$ & $D(\%)$ \\
\hline NLO, 540 & -8.284 & -28.03 & 65.2 & 0.62 & 0.08 & 6.00 \\
NLO, 560 & -8.091 & -26.91 & 68.2 & 0.68 & 0.09 & 6.41 \\
NLO, 580 & -7.847 & -25.55 & 72.2 & 0.76 & 0.10 & 6.84 \\
NLO, 600 & -7.546 & -23.96 & 77.7 & 0.86 & 0.11 & 7.30 \\
CD-Bonn & -8.012 & -27.05 & 77.6 & 0.48 & 0.22 & 10.72 \\
$\exp$ & -8.48 & -29.00 & $\cdots$ & $\cdots$ & $\cdots$ & $\cdots$ \\
\hline \hline
\end{tabular}

for $\Lambda=540$ and $\Lambda=600 \mathrm{MeV} / c$, respectively. The $d \sigma$ and $T_{2 k}$ agree rather well with the CD-Bonn result and thus with the data. We consider this to be an important result, demonstrating that the chiral $N N$ forces are very well suited to also describe low-energy $3 N$ scattering observables rather quantitatively. On top of that, the chiral force predictions are now significantly higher in the maxima of $A_{y}$ than for CD-Bonn and break the long-standing situation that all standard realistic $N N$ forces up to now underpredict the maxima by about $30 \%$. This is called the $A_{y}$ puzzle [21]. We are now in fact rather close to the experimental $n d$ values. Since we restrict ourselves to NLO we cannot expect a final answer from the point of view of chiral dynamics, but this result for $A_{y}$ is very interesting. In that context it is important to note that on a $2 N$ level the chiral potential predictions for the $n p A_{y}$ agree well with the ones based on the Nijmegen PSA and with the
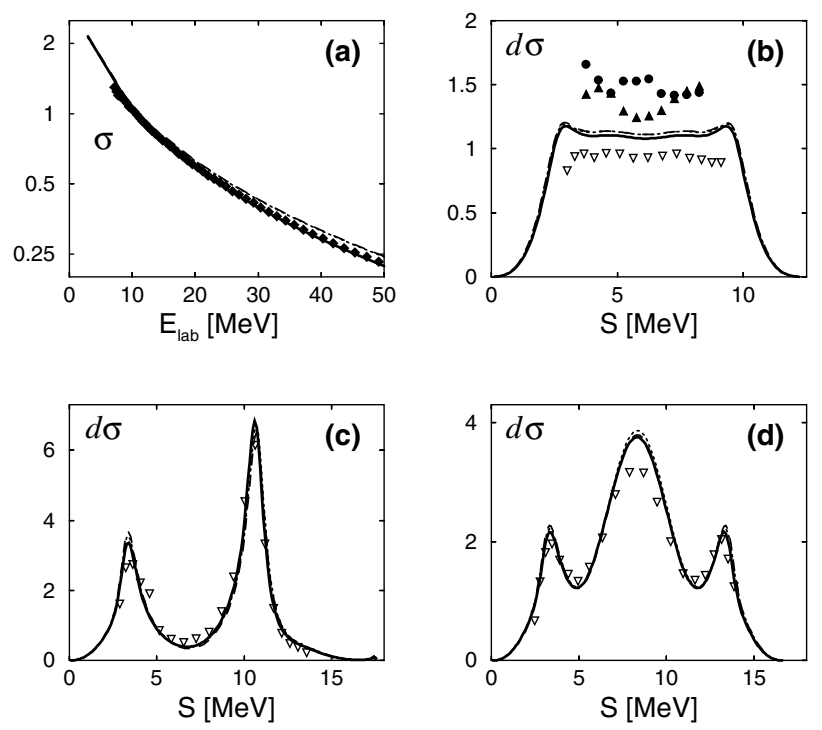

FIG. 1. (a) The $n d$ total cross section (in barns) for the chiral forces $(\Lambda=540 \mathrm{MeV} / c$, dotted curve; $\Lambda=600 \mathrm{MeV} / c$, dashed curve), and CD-Bonn (thick solid curve). (b)-(d) Chiral $N N$ force and CD-Bonn predictions for $n d$ breakup cross section $d \sigma$ at $E_{\text {lab }}=13 \mathrm{MeV}$ along the kinematical locus $S$. The various breakup configurations are chosen according to Figs. 42, 39 , and 35 in [21], respectively. $p d$ data are $(\nabla)$ [22]; $n d$ data are $(\mathbf{\Delta})$ [23], (○) [24], (४) [25].
CD-Bonn predictions as shown in Fig. 3. This agreement is especially important, since the $n p A_{y}$ is rather sensitive to the ${ }^{3} P_{j} N N$ phase shifts, which influence also strongly the $3 N A_{y}$.
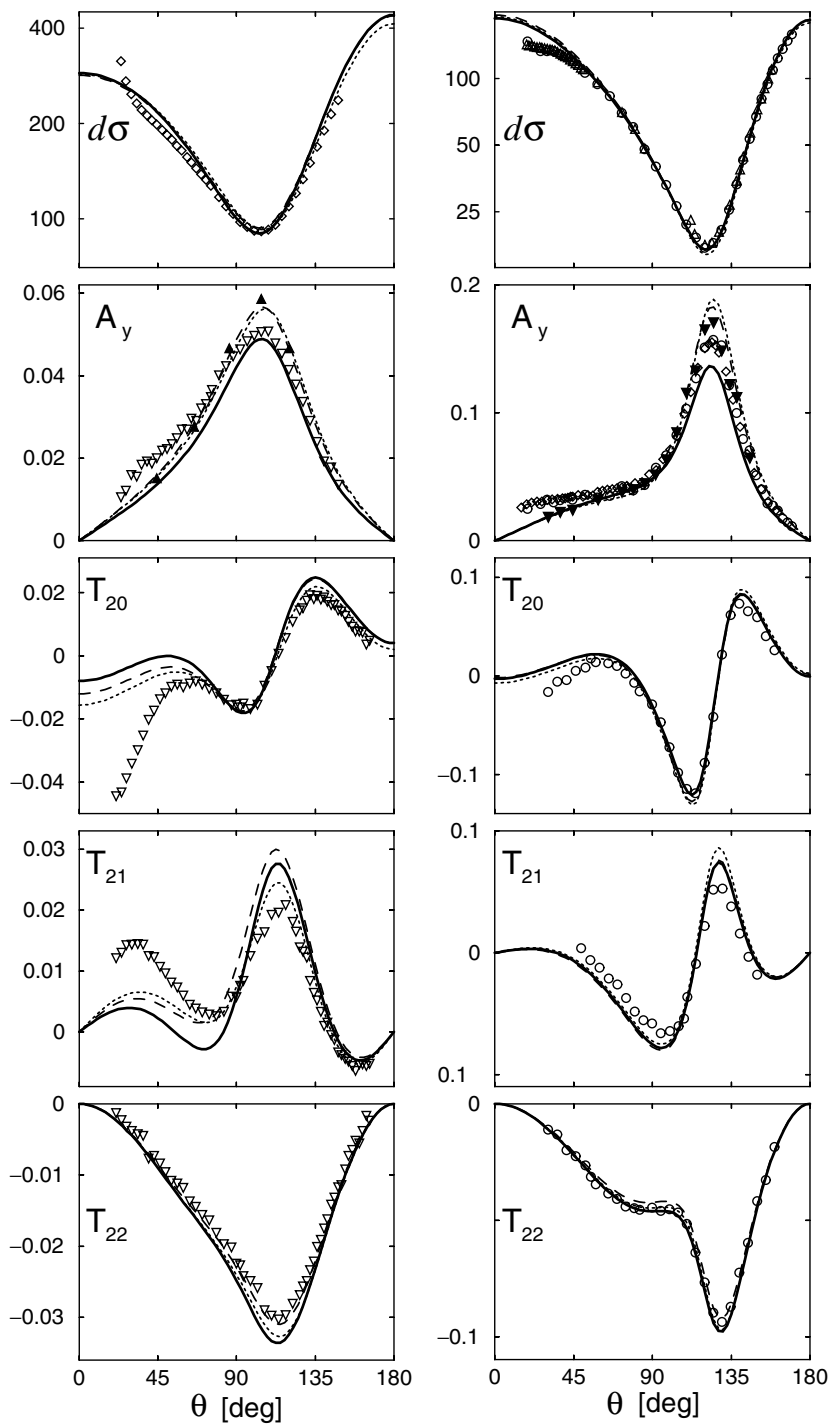

FIG. 2. nd elastic scattering observables at $E_{n}=3 \mathrm{MeV}$ (left column) and $E_{n}=10 \mathrm{MeV}$ (right column). pd data are $(\diamond)$ [27], $(\nabla)$ [28], $(\triangle)$ [29], $(\bigcirc)$ [30]. nd data are $(\mathbf{\Delta})$ [31], ( $)$ [32]. For remaining notations see Fig. 1. 

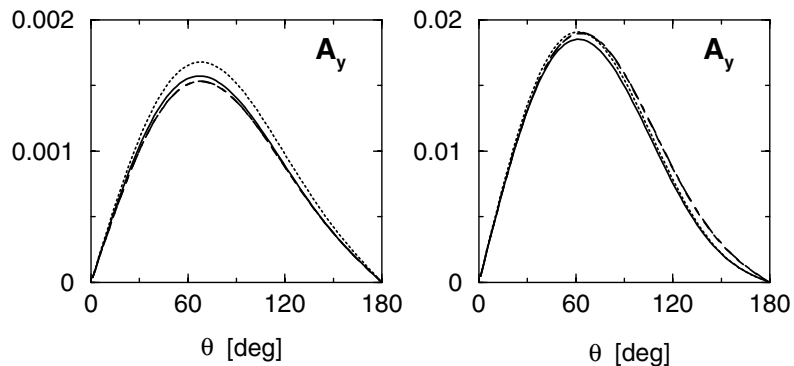

FIG. 3. nd analyzing powers $A_{y}$ at 3 (left panel) and $12 \mathrm{MeV}$ (right panel) for the chiral forces $(\Lambda=540 \mathrm{MeV} / c$, dashed curve; $\Lambda=600 \mathrm{MeV} / c$, dot-dashed curve), PSA (solid curve) and CD-Bonn (dotted curve).

These very first results using chiral $N N$ forces in $3 N$ and $4 N$ systems are very promising. In particular, scattering and breakup observables show a rather mild cutoff dependence. This indicates that these effective chiral forces are very well suited to describe also low-energy properties of nuclear systems beyond $A=2$. They agree rather well with standard nuclear force predictions as exemplified with CD-Bonn and most importantly they shed new light on the $A_{y}$ puzzle. Our result provides a counter example to the suggestion [34] that $N N$ forces alone cannot describe $2 N$ and $3 N A_{y}$ 's at the same time and 3NF's should cure the $3 N A_{y}$ puzzle. Examples for such trials can be found in [33,35]. We also note that the work of Refs. [35] and [36] indicates the possible existence of some large NNLO corrections going in the right direction. However, without a complete NNLO calculation, which includes these effects but also many others, it would be speculative to draw any premature conclusions. The study of the protons magnetic polarizability offers a stringent example - the large subleading effect due to the delta resonance which seemingly invalidates the leading pion loop results (which is in good agreement with the data) is almost entirely canceled by a subleading pion loop effect which could be found only in the complete NNLO calculation (see [37] for a detailed discussion).

It will be very interesting to perform the next step and use the NNLO NN forces, which is a systematic improvement. On this level also $3 N$ forces have to be incorporated the first time, which in $\chi \mathrm{PT}$ are defined consistently with the $N N$ force. It should be mentioned further that due to the underlying Lagrangian the coupling of external probes is well defined and exchange currents consistent with the nuclear forces can be generated easily.
This work was supported by the Deutsche Forschungsgemeinschaft (A.N.) and by the Polish Committee for Scientific Research under Grant No. 2P03B02818 (H. W.). The numerical calculations have been performed on the CRAY T90 and T3E of the John von Neumann Institute for Computing in Jülich, Germany.

[1] S. Weinberg, Phys. Lett. B 251, 288 (1990); Nucl. Phys. B363, 3 (1991).

[2] U. van Kolck, Phys. Rev. C 49, 2932 (1994).

[3] C. Ordóñez et al., Phys. Rev. C 53, 2086 (1996).

[4] T.-S. Park et al., Nucl. Phys. A646, 83 (1999).

[5] N. Kaiser et al., Nucl. Phys. A625, 758 (1997).

[6] D. B. Kaplan et al., Phys. Lett. B 424, 390 (1998).

[7] S. Fleming et al., Nucl. Phys. A677, 313 (2000).

[8] E. Epelbaum et al., Nucl. Phys. A637, 107 (1998).

[9] E. Epelbaum et al., Nucl. Phys. A671, 295 (2000).

[10] P. Büttiker et al., Nucl. Phys. A668, 97 (2000).

[11] A. Nogga et al., Phys. Rev. Lett. 85, 944 (2000).

[12] W. Glöckle et al., Phys. Rev. Lett. 71, 971 (1993).

[13] H. Kamada et al., Nucl. Phys. A548, 205 (1992).

[14] P. F. Bedaque et al., Phys. Rev. C 58, R641 (1998); Phys. Rev. Lett. 82, 463 (1999); Nucl. Phys. A676, 357 (2000).

[15] P. F. Bedaque et al., Nucl. Phys. A671, 357 (2000).

[16] For the potential parameters contact E. E.

[17] P. Lepage, nucl-th/9706029.

[18] V. G. Stocks et al., Phys. Rev. C 48, 792 (1993); NN-Online program, http://nn-online.sci.kun.nl.

[19] R. Machleidt et al., Phys. Rev. C 53, R1483 (1996).

[20] M. Walzl et al., nucl-th/0010019 [Nucl. Phys. A (to be published)].

[21] W. Glöckle et al., Phys. Rep. 274, 107 (1996).

[22] G. Rauprich et al., Nucl. Phys. A535, 313 (1991).

[23] J. Strate et al., Nucl. Phys. A501, 51 (1989).

[24] H. R. Setze et al., Phys. Lett. B 388, 229 (1996).

[25] W. P. Abfalterer et al., Phys. Rev. Lett. 81, 57 (1998).

[26] C. R. Howell et al., Nucl. Phys. A631, 692c (1998).

[27] K. Sagara et al., Phys. Rev. C 50, 576 (1994).

[28] S. Shimizu et al., Phys. Rev. C 52, 1193 (1995).

[29] G. Rauprich et al., Few-Body Syst. 5, 67 (1988).

[30] F. Sperisen et al., Nucl. Phys. A422, 81 (1984).

[31] J. E. McAninch et al., Phys. Lett. B 307, 13 (1993).

[32] C. R. Howell et al., Few-Body Syst. 2, 19 (1987).

[33] A. Kievsky, Phys. Rev. C 60, 034001 (1999).

[34] D. Hüber et al., Phys. Rev. C 58, 674 (1998).

[35] L. Canton et al., nucl-th/0006070.

[36] D. Hüber et al., nucl-th/9910034.

[37] V. Bernard et al., Int. J. Mod. Phys. E 4, 193 (1995). 